\title{
Risk factors of neuropathic pain after thoracic surgery
}

\author{
Takahiro Homma, Yoshinori Doki, Yutaka Yamamoto, Toshihiro Ojima, Yoshifumi Shimada, Naoya \\ Kitamura, Naoki Yoshimura
}

Department of General Thoracic and Cardiovascular Surgery, Graduate School of Medicine and Pharmaceutical Sciences, University of Toyama, Toyama, Japan

Contributions: (I) Conception and design: T Homma; (II) Administrative support: T Homma, Y Doki, N Yoshimura; (III) Provision of study materials or patients: All authors; (IV) Collection and assembly of data: All authors; (V) Data analysis and interpretation: T Homma; (VI) Manuscript writing: All authors; (VII) Final approval of manuscript: All authors.

Correspondence to: Takahiro Homma, MD. Department of General Thoracic and Cardiovascular Surgery, Graduate School of Medicine and Pharmaceutical Sciences, University of Toyama, 2630 Sugitani, Toyama 930-0194, Japan. Email: hommat@med.u-toyama.ac.jp.

\begin{abstract}
Background: This study aimed to clarify the incidence and risk factors of neuropathic pain after thoracic surgery, focusing especially on patients who underwent complete video-assisted thoracoscopic surgery (VATS). Methods: We retrospectively identified 185 patients who underwent thoracic surgery at our hospital over a 2-year period. Logistic regression analysis was used to analyze the association of various factors with postoperative neuropathic pain.

Results: Forty-eight (25.9\%) patients developed postoperative neuropathic pain, and 9 (18.8\%) of these patients reported persistent pain 1 year postoperatively. The median interval from surgical treatment to the onset of neuropathic pain was 7 days, and the duration was 50 days. Multivariate logistic regression analysis revealed a significant positive correlation between postoperative neuropathic pain and preoperative use of hypnotic medication [odds ratio (OR), 5.45; 95\% confidence interval (CI); 2.52-12.17] and duration of surgery $\geq 2.5$ hours (OR, 2.72; 95\% CI, 1.27-6.09), and a significant negative association with the complete VATS approach (OR, 0.18; 95\% CI, 0.073-0.42).

Conclusions: Preoperative use of hypnotic medication, the thoracotomy approach, and duration of surgery $\geq 2.5$ hours are associated with increased risk of neuropathic pain after thoracic surgery. The complete VATS approach could decrease the incidence of postoperative neuropathic pain, regardless of the duration of surgery.
\end{abstract}

Keywords: Neuropathic pain; thoracic surgery; video-assisted thoracic surgery; thoracotomy

Submitted Dec 31, 2017. Accepted for publication Apr 24, 2018.

doi: $10.21037 /$ jtd.2018.05.25

View this article at: http://dx.doi.org/10.21037/jtd.2018.05.25

\section{Introduction}

Neuropathic pain after thoracic surgery, known as postthoracotomy pain, is common and sometimes severe. The pain often becomes chronic and is associated with symptoms including burning, shooting, shocking, pressure-like, and aching sensations (1). Chronic pain can cause insomnia and loss of appetite and may incapacitate patients, making daily activities difficult or impossible (2). Although there are several mechanisms for the development of chronic pain after thoracotomy, intercostal nerve damage and subsequent dysfunction has long been implicated. Many methods of pain management have been tested, and video-assisted thoracoscopic surgery (VATS) is believed to result in less severe postoperative pain.

Our hospital began using complete VATS lobectomy without a mini-thoracotomy in 2002 (3). Since then, most patients requiring lobectomy at our hospital have undergone complete VATS. Complete VATS is a minimally invasive procedure, and patients are discharged from the hospital sooner compared with patients who undergo thoracotomy. However, some patients who often denied 
pain during the initial hospitalization return complaining of postoperative pain. Furthermore, the pain these patients describe is characteristic of neuropathic pain. Neuropathic pain can occur even after complete VATS without a minithoracotomy.

Neuropathic pain after thoracic surgery has been investigated previously, although many questions remain unanswered, including the onset, duration, location, and patient characteristics that may increase the risk of postoperative neuropathic pain. It is also unclear as to whether the incidence of neuropathic pain after complete VATS is equal to the incidence of VATS with a minithoracotomy. The purpose of this study was to determine the timing and perioperative risk factors of neuropathic pain after thoracic surgery, as well as whether the complete VATS approach has a lower incidence of postoperative neuropathic pain.

\section{Methods}

\section{Study design and patients}

In this retrospective study, we identified all patients older than 18 years who underwent thoracotomy or VATS at the Toyama University Hospital (Toyama, Japan) between January 2010 and December 2011. Exclusion criteria were as follows: previous history of thoracic surgery, preoperative neuropathic pain, or if a median sternotomy was performed. These dates were chosen to include all available data from our clinical database at the onset of this study. This study was approved by the Ethics Committee of the University of Toyama with a waiver of informed consent due to its retrospective design (RIN 29-52).

\section{Inclusion criteria for patients with neuropathic pain}

Neuropathic pain is defined as "pain caused by a lesion or disease of the somatosensory system" (4). We made use of validated neuropathic pain screening tools such as the Leeds assessment of neuropathic symptoms and signs and the related self-completed version to ensure consistency and reduce missing data between assessments in the acute and follow-up periods $(5,6)$. Chronic pain was defined as pain that persisted for more than three months after surgery.

\section{Surgical approach}

General anesthesia was performed using a single-lung ventilation technique with a double-lumen endotracheal tube. Patients were placed in a lateral decubitus position. The most common surgical approach in this study was complete VATS. In cases of partial resection, mediastinal tumor resection, pleural/chest wall resection, and decortication, we performed VATS with three 5-mm ports. For lobectomy and segmentectomy cases, we used an additional $5-\mathrm{mm}$ port and a subxiphoid incision to remove the resected lobe or lung segment (3). Patients with locally advanced carcinoma or cases in which a complex segmentectomy or partial resection of a deep-seated lung tumor was required typically underwent VATS with a minithoracotomy or an open thoracotomy. Three surgeons and their staff performed the surgeries, and complicated surgeries were performed by the chief surgeon. A 24-French chest tube was inserted at the end of each procedure.

\section{Pain management}

Figure 1 showed pain management algorithm. Standard perioperative pain control was performed using epidural analgesia, intravenous fentanyl, or oral analgesics. Epidural analgesia was considered to be the standard pain management technique. Intravenous fentanyl was administered to patients with contraindications for epidural analgesia, such as prolonged coagulation time, or patients who declined epidural analgesia.

Thoracic epidural catheterization was usually performed prior to anesthesia induction at the T4-5, T56, or T6-7 intervertebral space with a loss-of-resistance technique. Catheter placement was confirmed with a test dose of $4 \mathrm{~mL}$ of $1 \%$ lidocaine. The epidural infusion was started in the operating room. The drug combination in the continuous infusions was $2.0 \mathrm{mg} / \mathrm{mL}$ ropivacaine and $2.5 \mu \mathrm{g} / \mathrm{mL}$ fentanyl. The infusion rate was adjusted to between 2 and $4 \mathrm{~mL} / \mathrm{h}$.

In patients who did not received an epidural, intravenous fentanyl was started in the operating room. The fentanyl infusion rate was adjusted to between 0.01 and $0.04 \mathrm{mg} / \mathrm{h}$. Epidural analgesia or the intravenous fentanyl infusion was stopped on the day of chest tube removal. When a chest tube was unable to remove, epidural analgesia or the intravenous fentanyl infusion was stopped by the postoperative day 14 according to pain.

Oral analgesics mostly consisted of the non-steroidal anti-inflammatory drug loxoprofen $180 \mathrm{mg} /$ day beginning on the first postoperative day. In patients with an estimated glomerular filtration rate (eGFR) less than $50 \mathrm{~mL} / \mathrm{min}$, 


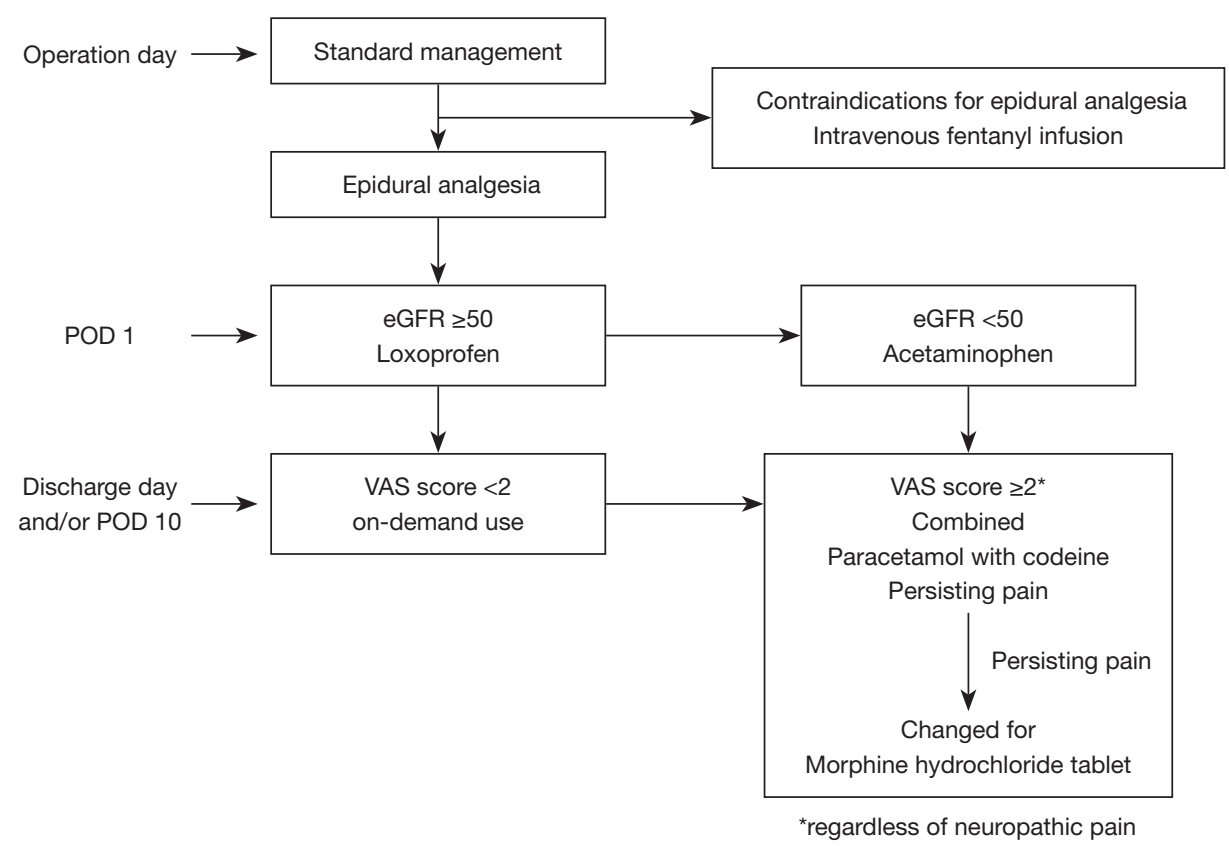

Figure 1 Postoperative pain management. POD, postoperative day; eGFR, estimated glomerular filtration rate; VAS, visual analog scale.

acetaminophen 1,200 mg/day was used in lieu of loxoprofen (7).

Pain was measured three times a day with the visual analog scale (VAS) during rest and coughing by floor nurses. The target level of pain control was a VAS score $<2$ during rest. When severe pain persisted beyond postoperative day 10 regardless of neuropathic pain, the patients were given oral opioids; the first choice was paracetamol with codeine, and the second choice was morphine hydrochloride tablet.

\section{Variables and outcomes}

The goal was to assess the perioperative risk factors of neuropathic pain after thoracic surgery. To do this, the following patient characteristics, surgical characteristics, and follow-up parameters were recorded: age, gender, smoking history, diabetes, body mass index (BMI), preoperative use of hypnotic medication, eGFR, diagnosis, diseased side, procedure type, surgical approach (complete VATS $v s$. thoracotomy), epidural analgesia, intraoperative blood loss, duration of surgery, chest tube duration, complications (prolonged air leak, defined as an air leak longer than 5 days, arrhythmia, pneumonia, delirium, acute respiratory distress syndrome, chylothorax), duration of postoperative hospitalization, postoperative acute pain requiring additional analgesics, number of analgesic doses used, postoperative neuropathic pain, and location and duration of postoperative neuropathic pain.

\section{Statistical analysis}

For the univariate analysis, differences between groups (i.e., patients who experienced neuropathic pain following surgery versus those who did not) were evaluated using a non-parametric Wilcoxon rank-sum test and either the $\chi^{2}$ test or Fisher's exact test for categorical variables when necessary. Significance was defined as $\mathrm{P}<0.05$.

Risk factors included in the multivariate analyses were selected on the basis of the univariate analyses, statistical independence, and clinical significance. Multivariate logistic regression was used to identify independent risk factors of neuropathic pain following thoracic surgery. A nominal logistic regression was initially performed with preoperative and intraoperative variables that were significant univariate predictors of the outcome being modeled. Due to the rarity of the outcome events being modeled, the variables identified in this step with a $\mathrm{P}$ value cutoff of 0.1 became candidates for the multivariate logistic regression models.

All reported $\mathrm{P}$ values are two-sided. The cutoff value for duration of surgery was set at 2.5 hours based on the results of a preliminary receiver operating characteristics analysis, which showed 153 minutes to be the optimal cutoff value with a C-index of 0.672. For intraoperative blood loss, $55 \mathrm{~mL}$ 
Table 1 Patient backgrounds

\begin{tabular}{|c|c|}
\hline Variable & Data \\
\hline Age, median [range] $\{\mathrm{IQR}\}$ & $68[15-87]\{57-76\}$ \\
\hline Gender, male/female & $126 / 59(68.1 / 31.9)$ \\
\hline Smoking history, yes/no & 98/87 (53.0/47.0) \\
\hline BMI, median (range) [IQR] & $\begin{array}{c}22.3(14.5-43.6) \\
{[19.9-24.8]}\end{array}$ \\
\hline Preoperative use of hypnotic medication & $53(28.6)$ \\
\hline eGFR (mL/min) & $76.1 \pm 21.2$ \\
\hline \multicolumn{2}{|l|}{ Diagnosis (\%) } \\
\hline Primary lung cancer & $105(56.8)$ \\
\hline Metastatic lung tumor & $21(11.4)$ \\
\hline Pneumothorax & $13(7.0)$ \\
\hline Other lung disease & $10(5.4)$ \\
\hline Mediastinal tumor & $16(8.6)$ \\
\hline Empyema & $6(3.2)$ \\
\hline Pleural/chest wall tumor & $14(7.6)$ \\
\hline Malignant disease (\%) & $133(71.9)$ \\
\hline Diseased side, right/left & $114 / 71(61.6 / 38.4)$ \\
\hline \multicolumn{2}{|l|}{ Procedure type (\%) } \\
\hline Partial resection & $38(20.5)$ \\
\hline Segmentectomy & $32(17.3)$ \\
\hline Lobectomy & $79(42.7)$ \\
\hline Mediastinal tumor resection & $16(8.6)$ \\
\hline Pleural/chest wall tumor resection & $14(7.6)$ \\
\hline Thoracostomy & $2(1.1)$ \\
\hline Decortication & $4(2.2)$ \\
\hline $\begin{array}{l}\text { Surgical approach, complete VATS/ } \\
\text { thoracotomy }\end{array}$ & 83/102 (44.9/55.1) \\
\hline Epidural analgesia (\%) & $163(88.1)$ \\
\hline $\begin{array}{l}\text { Intraoperative blood loss }(\mathrm{mL}) \text {, } \\
\text { median [range] }\{\mathrm{IQR}\}\end{array}$ & $80[1-2,800]\{1-225\}$ \\
\hline $\begin{array}{l}\text { Duration of surgery (min), } \\
\text { median [range] }\{\mathrm{IQR}\}\end{array}$ & $148[19-484]\{94-214\}$ \\
\hline $\begin{array}{l}\text { Chest tube duration (days), } \\
\text { median [range] }\{\mid \mathrm{QR}\}\end{array}$ & $2[0-62]\{1-4\}$ \\
\hline
\end{tabular}

Table 1 (continued)
Table 1 (continued)

\begin{tabular}{|c|c|}
\hline Variable & Data \\
\hline Complications (\%) & $52(28.1)$ \\
\hline Prolonged air leak & $22(11.9)$ \\
\hline Arrhythmia & $17(9.2)$ \\
\hline Pneumonia & $15(8.1)$ \\
\hline Delirium & $5(2.7)$ \\
\hline ARDS & $4(2.2)$ \\
\hline Chylothorax & $4(2.2)$ \\
\hline $\begin{array}{l}\text { Postoperative hospitalization (days), } \\
\text { median [range] }\{\mathrm{IQR}\}\end{array}$ & 9 [2-152] [7-12] \\
\hline $\begin{array}{l}\text { Postoperative acute pain requiring } \\
\text { additional analgesics (\%) }\end{array}$ & $98(53.0)$ \\
\hline $\begin{array}{l}\text { Number of analgesic uses, } \\
\text { median [range] }\{I Q R\}\end{array}$ & $2[1-41][1-4]$ \\
\hline Postoperative neuropathic pain (\%) & $48(25.9)$ \\
\hline
\end{tabular}

was also determined to be the cutoff value with a C-index of 0.744. All statistical analyses were performed with JMP software version 12.0 (SAS Institute, Inc., Cary, NC, USA).

\section{Results}

A total of 185 patients were enrolled in this study. The patient backgrounds are summarized in Table 1. Follow-up was completed in all patients within 12 months. Forty-eight (25.9\%) patients developed postoperative neuropathic pain, and $9(18.8 \%)$ of these patients reported pain that persisted one year postoperatively. The median interval from surgical treatment to neuropathic pain onset was 7 days, and the median duration of neuropathic pain was 50 days. All patients with neuropathic pain felt the pain along the anterior aspect of the surgical wound (including the nipple and epigastric region). No patients described neuropathic pain in other areas. Patients with postoperative neuropathic pain had significantly more severe acute postoperative pain that required additional analgesics $[35(72.9 \%)$ vs. 63 (46.0\%); $\mathrm{P}=0.0014$ ], higher frequency of analgesic use (4 vs. 
1; $\mathrm{P}<0.0001$ ), and longer postoperative hospitalization (11 vs. 8 days; $\mathrm{P}<0.0001)$.

\section{Univariate analysis of risk factors of neuropathic pain}

Table 2 showed the result of the univariate analysis of potential risk factors of postoperative neuropathic pain. The univariate analysis identified age $(\mathrm{P}=0.017)$, smoking history $(\mathrm{P}=0.0014)$, preoperative use of hypnotic medication $(\mathrm{P}<0.0001)$, primary lung cancer $(\mathrm{P}=0.0038)$, pneumothorax $(\mathrm{P}=0.023)$, malignant disease $(\mathrm{P}=0.016)$, partial resection $(\mathrm{P}=0.013)$, segmentectomy $(\mathrm{P}=0.047)$, complete VATS $(\mathrm{P}<0.0001)$, intraoperative blood loss $(\mathrm{P}<0.0001)$, duration of surgery $\geq 2.5$ hours $(\mathrm{P}=0.0023)$, and chest tube duration $(\mathrm{P}=0.0003)$ as significant risk factors of postoperative neuropathic pain.

\section{Multivariate analysis of risk factors of neuropathic pain}

Multivariate logistic regression analysis was performed using four independent variables for which the $\mathrm{P}$ values were $<0.10$ by univariate analysis. There was a significant positive correlation between neuropathic pain after thoracic surgery and preoperative use of hypnotic medication [odds ratio (OR), 5.45; 95\% confidence interval (CI), 2.52-12.17; $\mathrm{P}<0.0001$ ] and duration of surgery $\geq 2.5$ hours (OR, 2.72; 95\% CI, 1.27-6.09; $\mathrm{P}=0.0102)$. There was a significant negative correlation between neuropathic pain after thoracic surgery and undergoing complete VATS (OR, 0.18; 95\% CI, 0.073-0.42; $\mathrm{P}<0.0001$ ) (Table 3).

\section{Risk factor analysis of neuropathic pain following thoracic surgery in patients without epidural analgesia}

Subgroup analysis was carried out for the patients who did not receive epidural analgesia $(n=22)$. Age, intraoperative blood loss, and duration of surgery were identified as possible risk factors of neuropathic pain by the univariate analyses. Duration of surgery was significantly associated with age and intraoperative blood loss.

\section{Risk factor analysis of neuropatbic pain following thoracic surgery in patients with impaired renal function}

Subgroup analysis was carried out for the patients with an eGFR $<50 \mathrm{~mL} / \mathrm{min}(\mathrm{n}=17)$. Smoking history and preoperative use of hypnotic medication were identified as possible risk factors of neuropathic pain by the univariate analyses. Preoperative use of hypnotic medication was significantly associated with smoking history.

\section{Risk factor analysis of neuropathic pain following thoracic surgery in patients who underwent the complete VATS approach}

Subgroup analysis was carried out for the patients who underwent the complete VATS approach $(n=83)$. Preoperative use of hypnotic medication and intraoperative blood loss were identified as possible risk factors of neuropathic pain by the univariate analyses. There was no significant difference in the duration of surgery between the group that underwent complete VATS and the groups that underwent mini-thoracotomy or thoracotomy (153 vs. 128 minutes; $\mathrm{P}=0.31$ ). Multivariate analysis using these factors only identified preoperative use of hypnotic medication as an independent risk factor of postoperative neuropathic pain in patients who underwent complete VATS (OR, 5.98; 95\% CI, 1.29-32.89; $\mathrm{P}=0.022$ ) (Table 4).

\section{Risk factor analysis of neuropathic pain following thoracic surgery in patients who underwent the thoracotomy approach}

Subgroup analysis was carried out for the patients who underwent the thoracotomy or mini-thoracotomy approach $(n=102)$. Preoperative use of hypnotic medication, intraoperative blood loss, and duration of surgery were identified as possible risk factors of neuropathic pain by the univariate analyses. Duration of surgery was significantly associated with intraoperative blood loss.

Multivariate analysis using these factors identified preoperative use of hypnotic medication (OR, 4.97; 95\% CI, $1.99-13.11 ; \mathrm{P}=0.0005)$ and duration of surgery $\geq 2.5$ hours as independent risk factors of postoperative neuropathic pain (OR, 3.63; 95\% CI, 1.48-9.47; P=0.0045) (Table 5).

\section{Risk factor analysis of neuropatbic pain following thoracic surgery in patients who underwent lung resections}

Subgroup analysis was carried out for the patients who underwent lung resections ( $\mathrm{n}=149$ ). Smoking history, preoperative use of hypnotic medication, primary lung cancer, pneumothorax, partial resection, complete VATS, intraoperative blood loss, duration of surgery $\geq 2.5$ hours, and chest tube duration were identified as possible risk factors of neuropathic pain by the univariate analyses. Duration of surgery was significantly associated with 
Table 2 Univariate analysis of all study patients with postoperative neuropathic pain

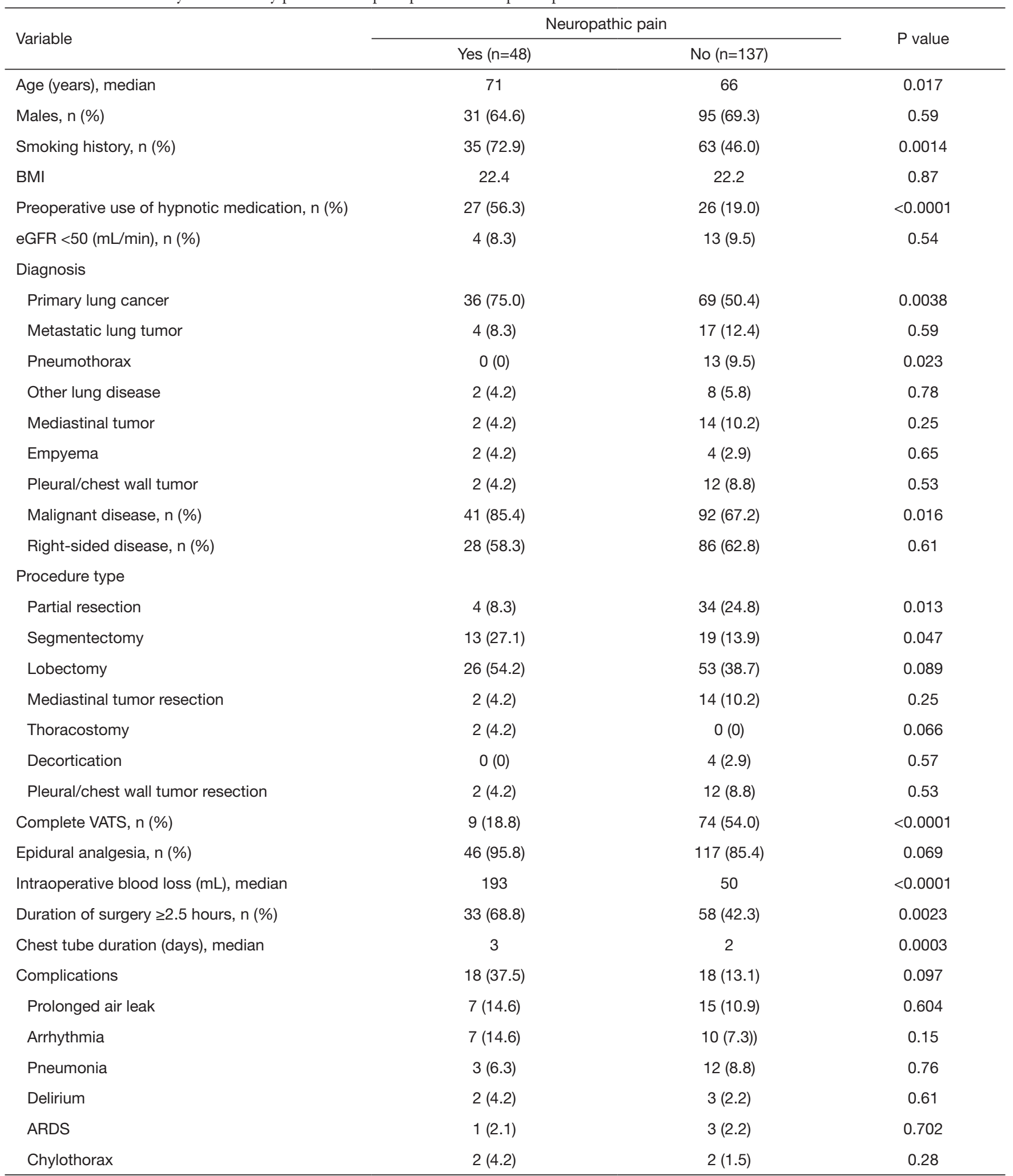

BMI, body mass index; eGFR, estimated glomerular filtration rate; VATS, video-assisted thoracoscopic surgery; ARDS, acute respiratory distress syndrome. 
Table 3 Multivariate logistic regression analysis of all study patients with postoperative neuropathic pain

\begin{tabular}{llcl}
\hline Variable & OR & $95 \% \mathrm{Cl}$ & $\mathrm{P}$ value \\
\hline Preoperative use of hypnotic medication & 5.45 & $2.52-12.17$ & $<0.0001$ \\
Complete VATS, $\mathrm{n}(\%)$ & 0.18 & $0.073-0.42$ & $<0.0001$ \\
Duration of surgery $\geq 2.5$ hours, $\mathrm{n}(\%)$ & 2.72 & $1.27-6.09$ & 0.0102 \\
\hline
\end{tabular}

OR, odds ratio; $\mathrm{Cl}$, confidence interval; VATS, video-assisted thoracoscopic surgery.

Table 4 Multivariate logistic regression analysis of the complete VATS subgroup

\begin{tabular}{lccc}
\hline Variable & OR & $95 \% \mathrm{Cl}$ & $\mathrm{P}$ value \\
\hline Preoperative use of hypnotic medication & 5.98 & $1.29-32.89$ & 0.022 \\
Intraoperative blood loss $\geq 55 \mathrm{~mL}$ & 3.48 & $0.67-25.9$ & 0.14 \\
\hline
\end{tabular}

OR, odds ratio; $\mathrm{Cl}$, confidence interval; VATS, video-assisted thoracoscopic surgery.

Table 5 Multivariate logistic regression analysis of the thoracotomy subgroup

\begin{tabular}{llll}
\hline Variable & OR & $95 \% \mathrm{Cl}$ & $\mathrm{P}$ value \\
\hline Preoperative use of hypnotic medication & 4.97 & $1.99-13.11$ & 0.0005 \\
Duration of surgery $\geq 2.5$ hours & 3.63 & $1.48-9.47$ & 0.0045 \\
\hline
\end{tabular}

OR, odds ratio; $\mathrm{Cl}$, confidence interval.

Table 6 Multivariate logistic regression analysis of lung resection subgroup

\begin{tabular}{lccc}
\hline Variable & OR & $95 \% \mathrm{Cl}$ & $\mathrm{P}$ value \\
\hline Preoperative use of hypnotic medication & 4.16 & $1.79-9.93$ & 0.0009 \\
Complete VATS, $\mathrm{n}(\%)$ & 0.22 & $0.070-0.58$ & 0.0018 \\
Duration of surgery $\geq 2.5$ hours, $\mathrm{n}(\%)$ & 2.65 & $1.12-6.61$ & 0.026 \\
\hline
\end{tabular}

OR, odds ratio; $\mathrm{Cl}$, confidence interval; VATS, video-assisted thoracoscopic surgery.

intraoperative blood loss.

Multivariate analysis using these factors identified preoperative use of hypnotic medication (OR, 4.16;95\% CI, 1.79-9.93; $\mathrm{P}=0.0009$ ) and duration of surgery $\geq 2.5$ hours as independent risk factors of postoperative neuropathic pain (OR, 2.65; 95\% CI, 1.12-6.61; $\mathrm{P}=0.026)$. There was a significant negative correlation between neuropathic pain after thoracic surgery and undergoing complete VATS (OR, 0.22; 95\% CI, 0.070-0.58; $\mathrm{P}=0.0018$ ) (Table 6).

Risk factor analysis of neuropathic pain following thoracic surgery in patients who underwent lung partial resection

Subgroup analysis was carried out for the patients who underwent lung partial resections $(\mathrm{n}=38)$. Age, preoperative use of hypnotic medication, primary lung cancer, intraoperative blood loss, and chest tube duration were identified as possible risk factors of neuropathic pain by the univariate analyses. Multivariate analysis using these factors identified just preoperative use of hypnotic medication (OR, 32.99; 95\% CI, 2.29-948.35; $\mathrm{P}=0.011$ ) (Table 7).

\section{Risk factor analysis of neuropathic pain following thoracic surgery in patients who underwent lobectomy}

Subgroup analysis was carried out for the patients who underwent lung partial resections $(n=142)$. Preoperative use of hypnotic medication, right-sided disease, complete 
Table 7 Multivariate logistic regression analysis of lung partial resection subgroup

\begin{tabular}{lccc}
\hline Variable & OR & $95 \% \mathrm{Cl}$ & $\mathrm{P}$ value \\
\hline Preoperative use of hypnotic medication & 32.9 & $2.29-948.35$ & 0.011 \\
\hline
\end{tabular}

OR, odds ratio; $\mathrm{Cl}$, confidence interval.

Table 8 Multivariate logistic regression analysis of lobectomy subgroup

\begin{tabular}{lccc}
\hline Variable & OR & $95 \% \mathrm{Cl}$ & $\mathrm{P}$ value \\
\hline Preoperative use of hypnotic medication & 4.20 & $1.43-13.24$ & 0.0009 \\
Complete VATS, $\mathrm{n}(\%)$ & 0.13 & $0.026-0.45$ & 0.0008 \\
\hline
\end{tabular}

OR, odds ratio; $\mathrm{Cl}$, confidence interval; VATS, video-assisted thoracoscopic surgery

VATS, intraoperative blood loss, and chest tube duration were identified as possible risk factors of neuropathic pain by the univariate analyses.

Multivariate analysis using these factors identified preoperative use of hypnotic medication (OR, 4.20; $95 \%$ CI, 1.43-13.24; $\mathrm{P}=0.0009)$ and complete VATS (OR, 0.13; 95\% CI, 0.026-0.45; $\mathrm{P}=0.0008$ ) (Table 8).

\section{Discussion}

Postoperative pain is associated with patient satisfaction with regard to both pain management and the overall hospital experience (8). Neuropathic pain following thoracic surgery is common, and it would behoove surgeons to better understand the potential risk factors of this potentially debilitating complication. Furthermore, neuropathic pain continues to occur despite increasing use of the less invasive VATS approach. This study sought to identify the perioperative risk factors of neuropathic pain after thoracic surgery, focusing especially on patients who underwent complete VATS. The results show that a quarter of patients experienced neuropathic pain after surgery; the median onset time was seven days, and the median duration of pain was 50 days. All of these patients felt the pain along the anterior aspect of the surgical wound and had significantly longer postoperative hospitalization than patients who did not experience neuropathic pain. The neuropathic pain persisted in about $20 \%$ of these patients one year after surgery. Our study found that risk factors of neuropathic pain after thoracic surgery include preoperative use of hypnotic medication, duration of surgery $\geq 2.5$ hours, and undergoing the thoracotomy approach. In particular, preoperative use of hypnotic medication and long duration of surgery ( $\geq 2.5$ hours) were risk factors in patients who did not receive epidural analgesia, patients with low renal function, and patients who underwent the complete VATS approach. The same tendencies were also denoted in patients underwent lung resection.

Postoperative neuropathic pain can result in prolonged hospitalization and increased healthcare cost. Most patients are treated with combinations of non-opioid analgesics. Opioids are key analgesic agents for treating moderate to severe pain after major surgery $(9,10)$. Some patients develop longer term persistent opioid use. Long term use of opioid is associated with increased risks of injury and cardiac events $(11,12)$. In particular, chronic neuropathic pain after thoracic surgery was an important determinant of prolonged opioid use (13). A better understanding of risk factors of neuropathic pain after thoracic surgery should greatly aid the decision making of both patients and clinicians.

Previous studies have investigated neuropathic pain after thoracic surgery $(14,15)$. However, few studies have included the onset, duration, and location of postoperative neuropathic pain, and whether there is a significant difference in the incidence of neuropathic pain in patients who undergo the complete VATS approach. The incidence of neuropathic pain after thoracic surgery has been reported to be $80 \%$ at three months and $61 \%$ at one year after surgery (15); however, our results suggest a lower incidence of neuropathic pain compared with previous reports. We believe that complete VATS is less invasive than either an open thoracotomy or VATS with a minithoracotomy. On the other hand, a prospective randomized study did not demonstrate any significant difference in pain and post-thoracotomy syndrome between muscle-sparing thoracotomy and posterolateral thoracotomy (16). So that, analysis between mini-thoracotomy and thoracotomy 
may not have much meaning. In all of the patients with neuropathic pain in this study, the pain manifested along the anterior aspect of the surgical wound. Most patients with chronic pain after thoracotomy also experienced almost same area of pain (15). This area is innervated by the anterior cutaneous branch of the intercostal nerves (2). In the present study, pain in areas innervated by other branches was not encountered; thus, these findings could help to distinguish neuropathic from non-neuropathic pain.

In this study, we found two additional risk factors of neuropathic pain after thoracic surgery: (I) preoperative use of hypnotic medication and (II) long duration of surgery ( $\geq 2.5$ hours), apart from the surgical approach and pain management technique. Psychosocial condition is a well-established factor affecting the perception and effects of chronic neuropathic pain conditions, including the presence of anxiety and depression, a diagnosis of malignant disease, the lack of a social support network, and poor social status (17-19). Preoperative fear and anxiety have been shown to be correlated with acute postoperative pain and analgesic consumption, and risks of subsequent persistent postoperative pain (20-22). Hypnotic drugs may be used more frequently in patients with anxiety and depression; therefore, we investigated the association between neuropathic pain and preoperative use of hypnotic medication. As expected, the preoperative use of hypnotic medication was a significant risk factor of postoperative neuropathic pain, regardless of the surgical approach or pain management technique. Thus, preoperative use of hypnotic medication could be a very useful predictor of neuropathic pain after thoracic surgery.

Long duration of surgery ( $\geq 2.5$ hours) is another reasonable risk factor of neuropathic pain. Thoracic surgery routinely crushes the intercostal nerves, particularly where the nerves are exposed along the caudal side of the rib (2). Intercostal nerve damage might increase proportionately with the duration of surgery. However, the duration of surgery showed no significant effect on the incidence of postoperative neuropathic pain in patients who underwent the complete VATS approach. Thus, the complete VATS approach could minimize intercostal nerve damage, regardless of the duration of surgery.

\section{Limitations}

This study has several limitations. First, the study population included only a limited number of consecutive cases at a single institution. Second, there may have been case selection bias regarding the surgical approach, as the choice between complete VATS or thoracotomy was based partly upon the surgeon's preference.

Postoperative neuropathic pain may be unavoidable for some high-risk patients after thoracic surgery. For most patients, however, prevention of postoperative neuropathic pain can yield a better postoperative experience, shorter hospitalization, and reduced healthcare cost. Pregabalin has become a validated drug for treating neuropathic pain in recent years $(9,23)$. and a randomized controlled prospective study should be carried out to determine if pregabalin is useful in preventing neuropathic pain after thoracic surgery.

\section{Conclusions}

Preoperative use of hypnotic medication, the thoracotomy surgical approach, and duration of surgery $\geq 2.5$ hours are significantly associated with neuropathic pain after thoracic surgery. The complete VATS approach could decrease the incidence of postoperative neuropathic pain, regardless of the duration of surgery.

\section{Acknowledgements}

We offer our sincere gratitude to Dr. Takehisa Asahi (Division of Anesthesiology, Joetsu General Hospital) for his valuable input in the preparation of this manuscript.

\section{Footnote}

Conflicts of Interest: The authors have no conflicts of interest to declare.

Ethical Statement: This study was approved by the Ethics Committee of the University of Toyama with a waiver of informed consent due to its retrospective design (RIN 29-52).

\section{References}

1. Butler S, Jonzon B, Branting-Ekenback C, et al. Predictors of severe pain in a cohort of 5271 individuals with selfreported neuropathic pain. Pain 2013;154:141-6.

2. Gerner P. Postthoracotomy pain management problems. Anesthesiol Clin 2008;26:355-67.

3. Doki $Y$, Ichiki K, Tsuda $M$, et al. Complete port-accessed lobectomy by the muscle-sparing method. Ann Thorac Surg 2004;77:2230-1.

4. Jensen TS, Baron R, Haanpaa M, et al. A new definition of 
neuropathic pain. Pain 2011;152:2204-5.

5. Bennett M. The LANSS Pain Scale: the Leeds assessment of neuropathic symptoms and signs. Pain 2001;92:147-57.

6. Bennett MI, Smith BH, Torrane N, et al. The S-LANSS score for identifying pain of predominantly neuropathic origin: validation for use in clinical and postal research. J Pain 2005;6:149-58.

7. Matsuo S, Imai E, Horio M, et al. Revised equations for estimated GFR from serum creatinine in Japan. Am J Kidney Dis 2009;53:982-92.

8. Maher DP, Wong W, Woo P, et al. Preoperative factors associated with HCAHPS responses of 2,758 surgical patients. Pain Med 2015;16:791-801.

9. Dahl JB, Nielsen RV, Wetterslev J, et al. Post-operative analgesic affects of paracetamol, NSAIDs, glucocorticoids, gabapentinoids, and their combinations: a topical review. Acta Anaesthesiol Scand 2014;58:1165-81.

10. Miguel R, Hubbell D. Pain management and spirometry following thoracotomy: a prospective, randomized study of four techniques. J Cardiothorac Vasc Anesth 1993;7:529-34.

11. Alam A, Gomes T, Zheng H, et al. Long-term analgesic use after low-risk surgery: a retrospective cohort study. Arch Intern Med 2012; 172:425-30.

12. Solomon DH, Rassen JA, Glynn RJ, et al. The comparative safety of opioids for nonmalignant pain in older adults. Arch Intern Med 2010; 170:1979-86.

13. Clarke H, Sonja N, Ko DT, et al. Rates and risk factors for prolonged opioid use after major surgery: population based cohort study. BMJ 2014;11:348:g1251.

14. Searle RD, Simpson MP, Simpson KH, et al. Can chronic neuropathic pain following thoracic surgery be predicted during the postoperative period? Interact Cardiovasc Thorac Surg 2009;9:999-1002.

Cite this article as: Homma T, Doki Y, Yamamoto Y, Ojima T, Shimada Y, Kitamura N, Yoshimura N. Risk factors of neuropathic pain after thoracic surgery. J Thorac Dis 2018;10(5):2898-2907. doi: 10.21037/jtd.2018.05.25
15. Perttunen K, Tasmuth T, Kalso E. Chronic pain after thoracic surgery: a follow-up study. Acta Anaesthesiol Scand 1999;43:563-7.

16. Elshiekh MA, Lo TT, Shipolini AR, et al. Does musclesparing thoracotomy as opposed to posterolateral thoraocotomy result in better recovery? Interact Cardiovasc Thorac Surg 2013;16:60-7.

17. Taenzer P, Melzack R, Jeans ME. Influence of psychological factors on postoperative pain, mood and analgesic requirements. Pain 1986;24:331-42.

18. Dualé C, Ouchchane L, Schoeffler P, et al. Neuropathic aspects of persistent postsurgical pain: a French multicenter survey with a 6-month prospective follow-up. J Pain 2014;15:24.e1-24.e20.

19. Schou Bredal I, Smeby NA, Ottesen S, et al. Chronic pain in breast cancer survivors: comparison of psychosocial, surgical, and medical characteristics between survivors with and without pain. J Pain Symptom Manage 2014;48:852-62.

20. Katz J, Buis T, Cohen L. Locked out and still knocking: predictors of excessive demands for postoperative intravenous patient-controlled analgesia. Can J Anaesth 2008;55:88-99.

21. Forsythe ME, Dunbar MJ, Henningar AW, et al. Prospective relation between catastrophizing and residual pain following knee arthplasty: two-year follow-up. Pain Res Manag 2008;13:335-41.

22. Sullivan M, Tanzer M, Stanish $W$, et al. Psychological determinants of problematic outcomes following total knee arthplasty. Pain 2009;143:123-9.

23. Mishriky BM, Waldron NH, Habib AS. Impact of pregabalin on acute and persistent postoperative pain: a systematic review and meta-analysis. $\mathrm{Br} \mathrm{J}$ Anaesth 2015;114:10-31. 